\title{
COMET Phase II
}

\section{Ajit Kurup*Imperial College London}

E-mail: a.kurup@imperial.ac.uk

The COMET experiment aims to search for muon to electron conversion with a sensitivity of $<10^{-17}$. The experiment will be built in two phases, with Phase-I aiming at a sensitivity of $<10^{-15}$ using a cylindrical drift chamber. The design of COMET Phase-II has a longer beam line to improve the quality of the muon beam and perform momentum selection of electrons. The detector system will include a straw tracker and a calorimeter, which will be prototyped and tested at Phase-I.

Staging the experiment is very important as this allows important measurements of the beam that will lead to a better understanding of backgrounds and the muon yield. These measurements can then be used to optimise the design of COMET Phase-II and will provide an improved estimate of the sensitivity of the experiment. The construction of Phase-I already underway and data taking will start in 2016 and Phase-II is scheduled to start data taking in 2019.

16th International Workshop on Neutrino Factories and Future Neutrino Beam Facilities 25 -30 August, 2014

University of Glasgow, United Kingdom

${ }^{*}$ Speaker. 


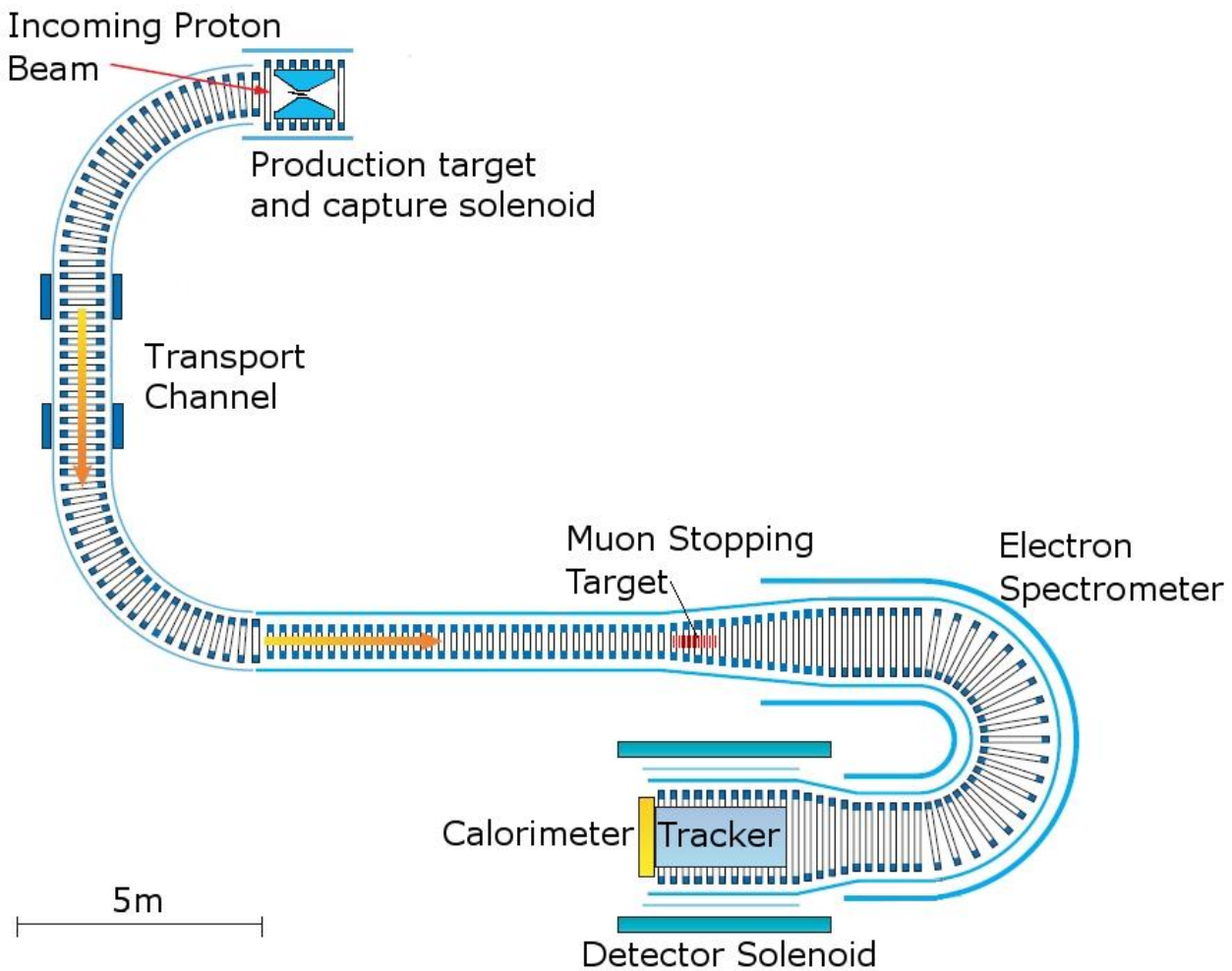

Figure 1: Schematic diagram of COMET Phase-II.

\section{Introduction}

The observation of neutrino oscillations shows that lepton flavour number is not conserved in the neutral lepton sector of the Standard Model (SM). This leads us to consider lepton flavour number violation in the charged lepton sector, e.g. $\mu^{-}+N(A, Z) \rightarrow e^{-}+N(A, Z)$. Developments in accelerator technology allow for significantly higher beam intensities than was previously achievable, together with the single particle final state, make $\mu^{-}+N(A, Z) \rightarrow e^{-}+N(A, Z)$ a particularly attractive mode to look for new physics.

The COherent Muon to Electron Transition (COMET) Experiment, based at J-PARC in Japan, aims to achieve a single event sensitivity of $2.6 \times 10^{-17}$. The COMET experiment will be constructed in two stages, with the ability to search for muon to electron conversion in both stages. COMET Phase-I will allow performance measurements of the beam line, which will be very important to understand sources of backgrounds for COMET Phase-II and it will be used to prototype the detector technology for Phase-II.

Figure 1 is a schematic diagram of the COMET experiment showing: the pion production target; the continuous superconducting solenoid transport channel; the muon stopping target, which is a series of aluminium disks; the electron spectrometer and the detector systems.

The material present here has been taken from the COMET conceptual design report [1]. Some specific details will have changed based on the progress made with the design of Phase-I and the Phase-II design will continue to evolve based upon experimental experience with Phase-I. 


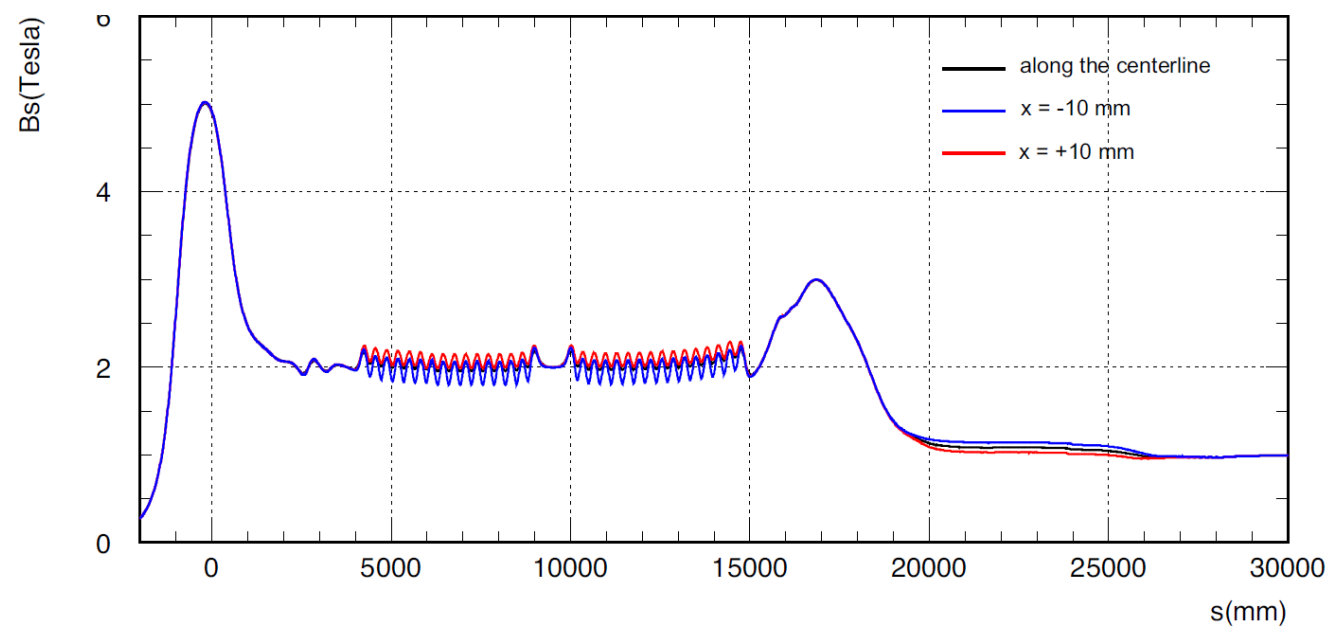

Figure 2: Magnitude of the field along the length of the beam line.

\section{The COMET Beam Line}

The bulk of the experiment is the continuous solenoid channel that is used to transport the beam to the detector region, while performing charge and momentum selection. The beam line also needs to be long enough to reduce the pion contamination. The requirements of the beam line are

- the muon transport should be long enough for pions to decay to muons. For instance, for about 20 meters, the pion survival rate for pions with the reference momentum is about $2 \times 10^{-3}$,

- the muon transport should have a high transport efficiency for muons with a momentum of $40 \mathrm{MeV} / \mathrm{c}$, and

- the muon transport should select muons with low momentum and eliminate muons of high momentum $\left(P_{\mu}>75 \mathrm{MeV} / \mathrm{c}\right)$ to avoid backgrounds from muon decays in flight.

Figure 2 shows the magnitude of the field along the length of the solenoid. This peaks at $5 \mathrm{~T}$ at the production target, drops down to $2 \mathrm{~T}$ in the bent parts of the solenoid, and then drops to $1 \mathrm{~T}$ in the detector region. The bent solenoid field provides momentum selection as charged particles will drift in the vertical direction and be collimated. The magnitude of the drift is given by

$$
\text { drift }=\frac{1}{q B}\left(\frac{s}{R}\right) \frac{P_{L}^{2}+\frac{1}{2} P_{T}^{2}}{P_{L}}
$$

This means that negative muons with the desired momentum of $40 \mathrm{MeV} / \mathrm{c}$ will also drift. In order to ensure that these stay on-axis a compensating dipole field is applied:

$$
B_{\text {comp }}=\frac{1}{q R} \frac{P_{L}^{2}+\frac{1}{2} P_{T}^{2}}{P_{L}}
$$

Figure 3 shows the magnitude of the dipole field. Figures 4 and 5 show the momentum distribution and dispersion of the beam at the stopping target using this configuration. The expected number of electrons from pion decay in the signal region is $<10^{-4}$. 


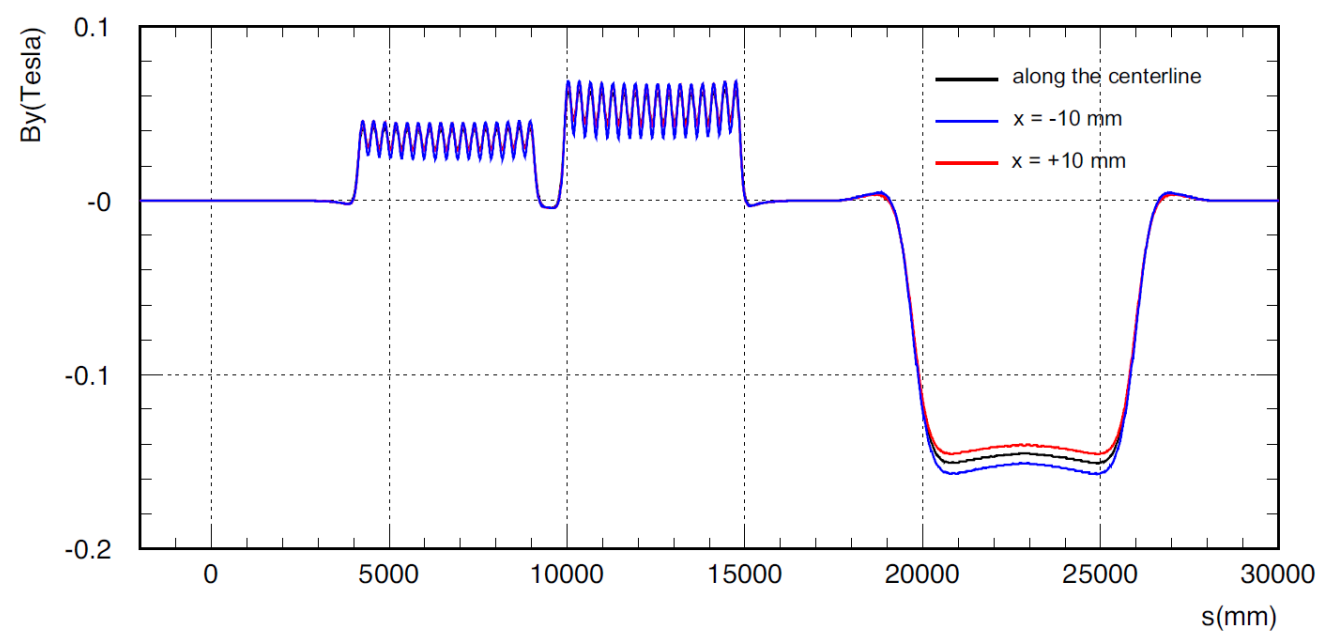

Figure 3: Magnitude of the compensating dipole field along the length of the beam line.

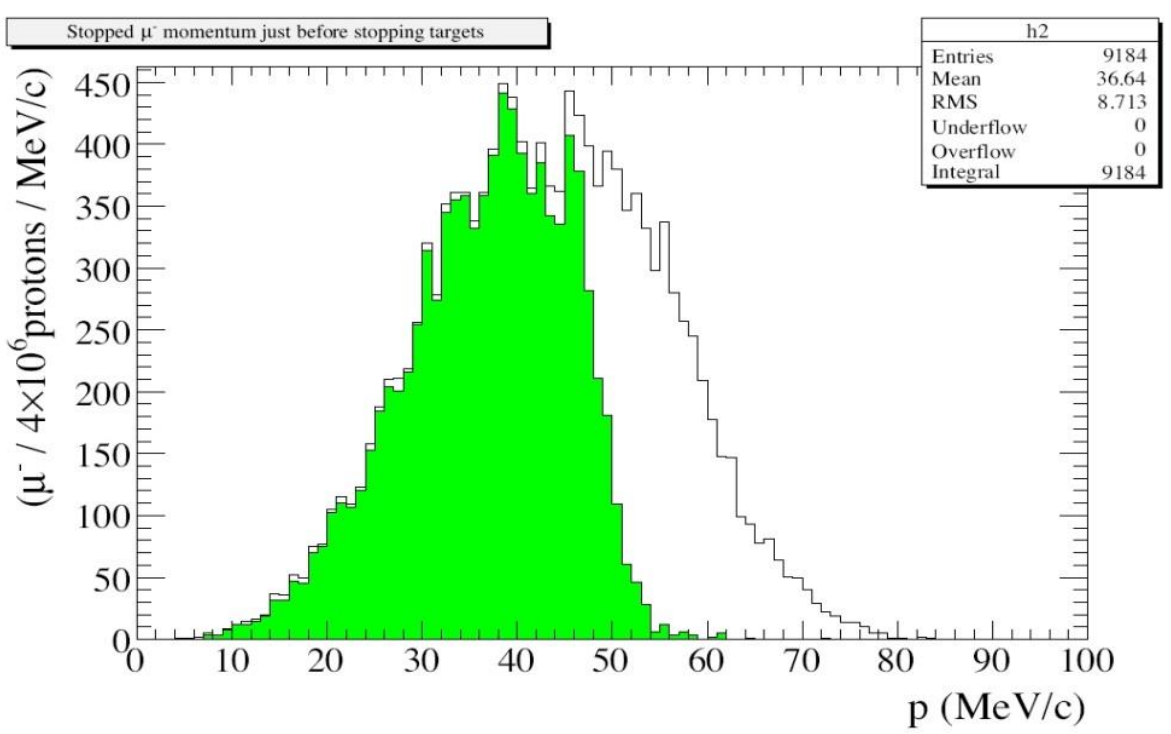

Figure 4: Momentum distribution of muons just before the stopping target. The region shaded in green shows the muons that are stopped by the target.

\subsection{The Electron Spectrometer}

The vertical dispersion of the electron spectrometer removes electrons with $\mathrm{P}<60 \mathrm{MeV} / \mathrm{c}$ and significantly reduces the trigger rate to $\sim 1 \mathrm{kHz}$. Figure 6 gives the decay-in-orbit spectrum and shows the fraction removed by the electron spectrometer. The transmission efficiency for electrons as a function of total energy is given in figure 7.

\section{The Straw Tracker}

The requirements of the tracker are: be able to operate in a vacuum and a $1 \mathrm{~T}$ solenoid field; 


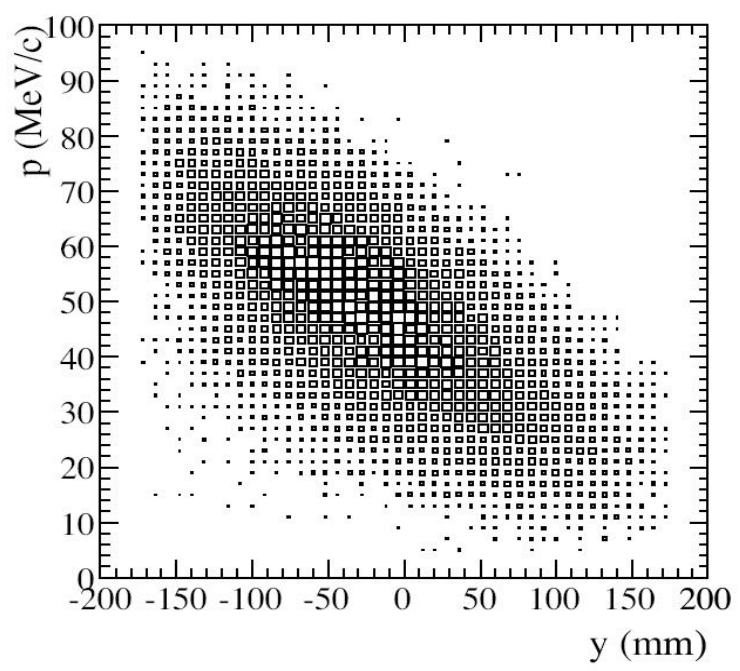

Figure 5: Dispersion at the end of the muon transport channel.

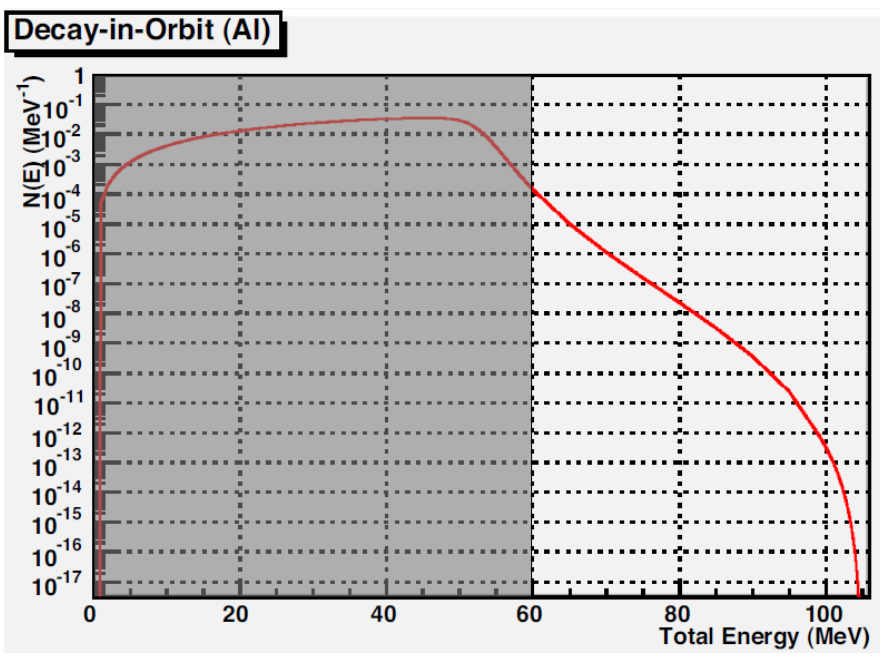

Figure 6: The decay-in-orbit rate as a function of electron energy. The shaded region indicated the electrons removed by the electron spectrometer.

be able to handle an $800 \mathrm{kHz}$ charged particle rate and an $8 \mathrm{MHz}$ gamma rate; and have a $0.4 \%$ momentum and a $700 \mu \mathrm{m}$ spatial resolution.

The tracker will be composed of 5 planes $48 \mathrm{~cm}$ apart with 2 views ( $\mathrm{x}$ and $\mathrm{y}$ ) per plane and 2 layers per view (staggered by one straw radius), see figure 8 . The gas filled straws have a diameter of $9.75 \mathrm{~mm}$ and are made from $12 \mu \mathrm{m}$ thick metalised polyimide with a gold coated tungsten anode wire. The tubes are produced by ultra-sonic welding at JINR.

Simulations of the straw tube have been performed using GARFIELD and the results are shown in figure 9 and prototyping of this design is underway, see figure 10. 


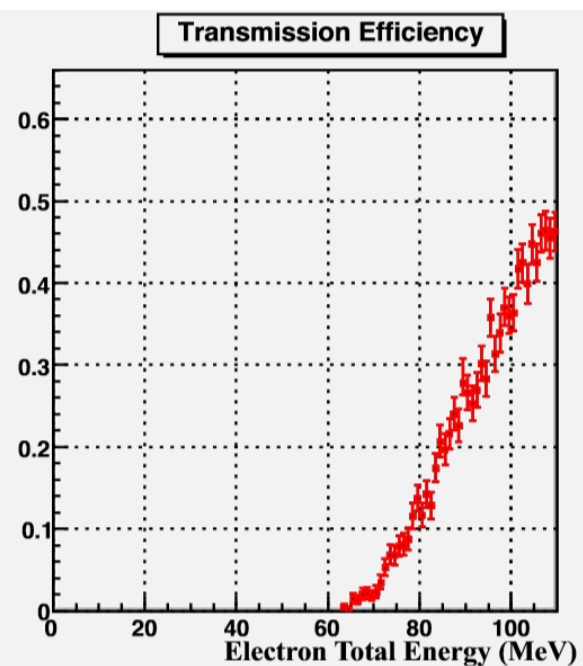

Figure 7: Transmission efficiency of the electron spectrometer as a function of electron energy.

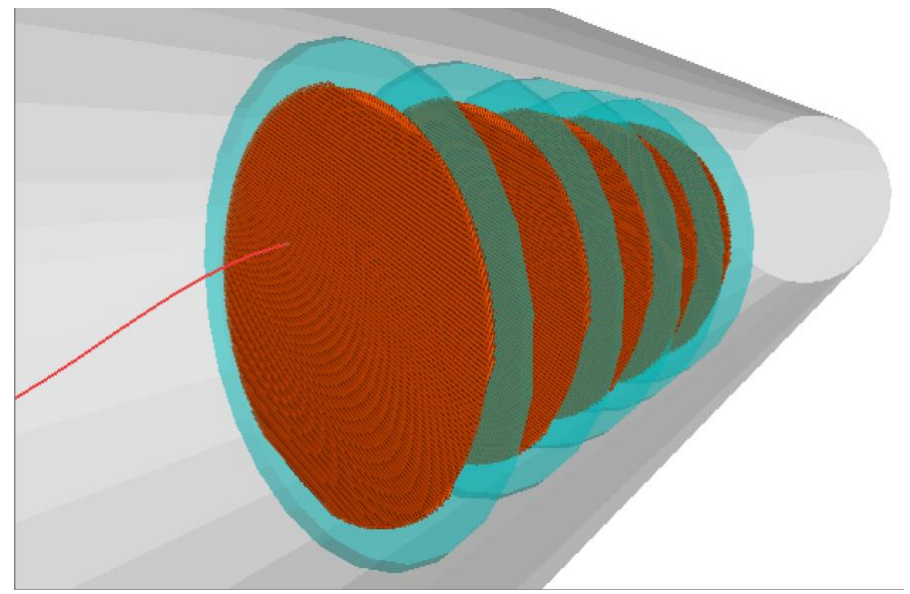

Figure 8: Schematic diagram of the straw tracker showing the five planes.

\section{The Calorimeter}

The requirements of the calorimeter are:

- Measure energy, PID and give additional position information. Can be used to make a trigger decision.

- $5 \%$ energy and $1 \mathrm{~cm}$ spatial resolution at $100 \mathrm{MeV}$.

- High segmentation ( $2 \times 2 \mathrm{~cm} 2$ cross-section).

- Operation in 1T magnetic field.

Two candidate inorganic scintillator materials were considered: Cerium-doped Lutetium Yttrium Orthosilicate (LYSO); and Cerium-doped Gd2SiO5 (GSO). The readout will be done using 

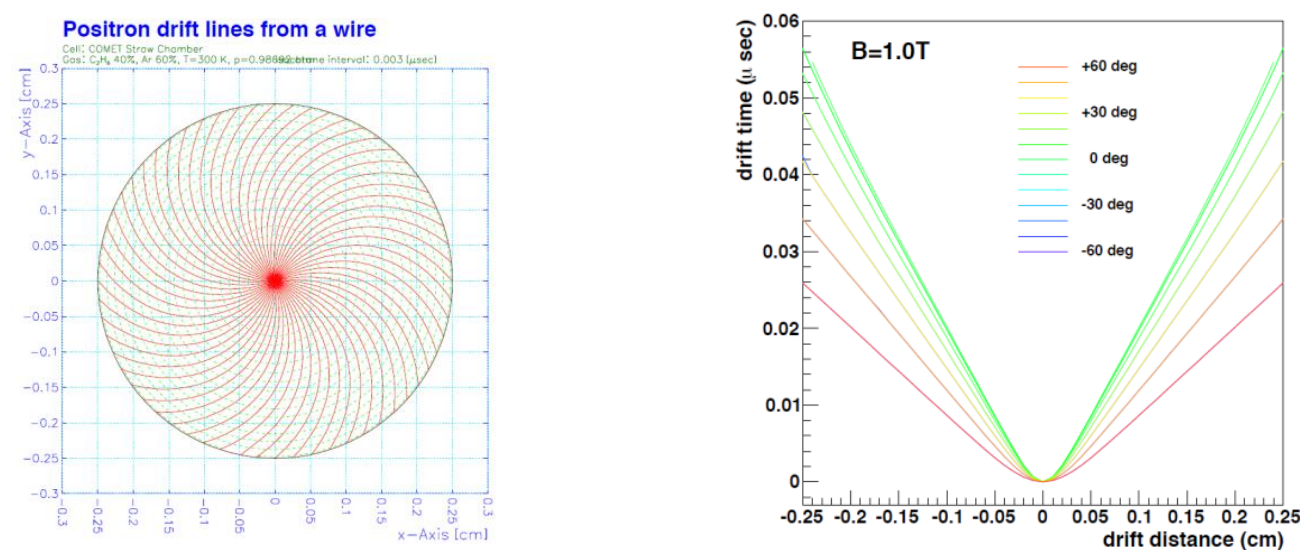

Figure 9: GARFIELD simulation of the drift lines and isochrones of a straw tube (left). Drift time (determined by GARFIELD simulations) as a function of distance from the anode wire for different incident angles (right).

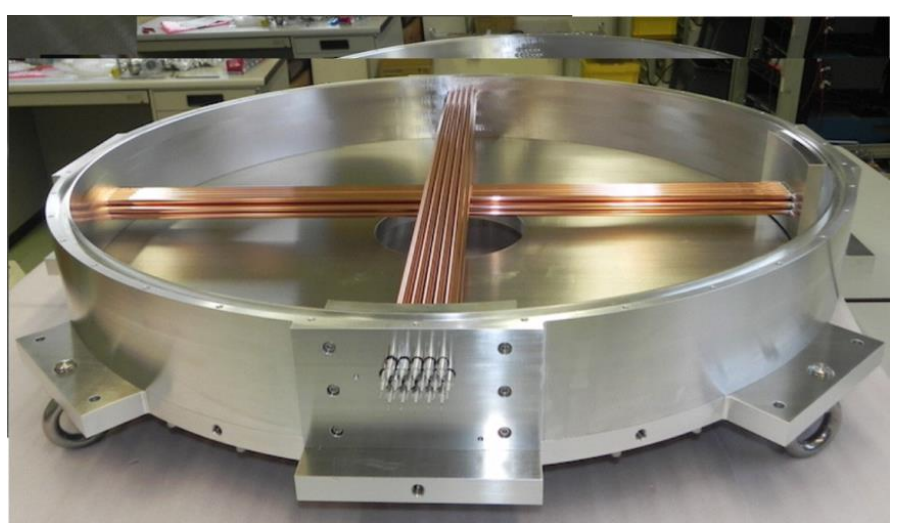

Figure 10: Prototype vessel with straw tube modules.

APD photo detectors with custom pre-amps. Beam tests have been performed of these crystals to determine their performance and based on this the decision to use LYSO was made.

\section{Measurements with Phase-I}

Building COMET in two phases provides the important opportunity to test many aspects of the beam line and detector technology with Phase-I. The types of measurements that are being considered fall into two categories: beam characterisation; and simulation validation. It is important to be able to characterise the beam as the continuous solenoid channel provides very little space for beam diagnostics. Simulation validation is important, in particular since there are large discrepancies between different hadron production models and very little experimental data. 

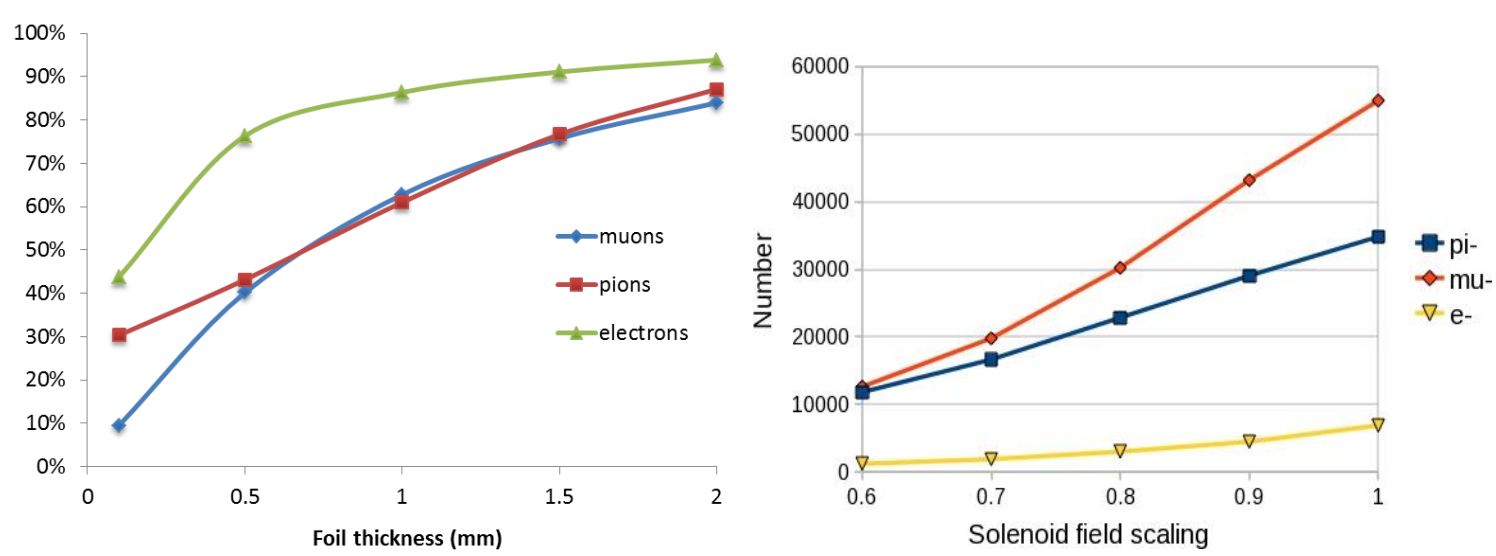

Figure 11: Effect on beam composition for different absorber foil thicknesses (left). Effect on beam composition for different solenoid field scalings (right).

It may be possible to investigate the composition of the beam using a simple detector and by varying the magnetic field and introducing different absorber materials in the beam line. Figure 11 shows the effect of foil thickness and solenoid field on beam composition.

\section{Summary}

Staging the COMET experiment will lead to an improved final design and Phase-I can provide several important measurements that will improve our understanding of backgrounds and hence improve the sensitivity of the experiment. Phase-II will also benefit from being able to test prototypes of detectors with a realistic beam.

Construction of Phase-II will start in 2017 and data taking will follow in 2019.

\section{References}

[1] The COMET Collaboration, "Conceptual design report for experimental search for lepton flavor violating $\mu^{-} \rightarrow e^{-}$conversion at sensitivity of $10^{-16}$ with a slow-extracted bunched proton beam (COMET)", KEK-2009-10, (2009). 